\title{
VARIACIONES RECIENTES DE LA TEMPERATURAS EN ESPAÑA: EL EFECTO DEL PERIODO ELEGIDO EN LAS TENDENCIAS DE LAS SERIES ESTACIONALES DE PROMEDIOS DE MÁXIMAS Y MÍNIMAS
}

\author{
José Carlos GONZALEZ-HIDALGO ${ }^{1,2}$, Dhais PEÑA-ANGULO ${ }^{1,2}$, \\ Celia SALINAS ${ }^{1,2}$, Azucena JIMÉNEZ-CASTAÑEDA ${ }^{1,2}$, MICHELE BRUNETTI $^{3}$ \\ ${ }^{1}$ Departamento de Geografia, Universidad de Zaragoza. \\ ${ }^{2} I U C A$, Universidad de Zaragoza. \\ ${ }^{3}$ ISAC-CNR, Bologna. \\ jcgh@unizar.es
}

\section{RESUMEN}

En una serie temporal de datos el signo de su tendencia, su significación y su tasa varían según el periodo seleccionado. Este hecho tiene gran importancia en el análisis climático para poder comparar diferentes investigaciones, y relevancia actual en el debate referido al "hiato térmico". En este trabajo se presenta un análisis de las variaciones de la tendencia de las temperaturas estacionales promedio de las máximas y mínimas de España peninsular (1951-2010) procedente de la base de datos MOTEDAS. La significación de la tendencia se evalúa con el test de Mann-Kendal, y la tasa con el estadístico de Sen. Los resultados muestran la ausencia de tendencias significativas recientes en invierno y otoño en máximas (Tmax) y mínimas (Tmin). En el mismo periodo la tendencia de Tmax en primavera no es significativa, mientras Tmin ha continuado siéndolo. Las tendencias de verano de Tmax y Tmin no son significativas desde al menos hace dos décadas. Las tasas de Tmax y Tmin de primavera y verano, las dos estaciones donde el aumento térmico ha sido más notable, son decrecientes en las décadas más recientes y en general la tasa de Tmin ha superado a la de Tmax desde inicios de la década de 1990.

Palabras clave: Temperaturas, Tendencias, ventanas temporales, España.

\begin{abstract}
In a temporal data series the signal of trend, intensity and significance vary accordingly selected period, and this is especially true in climate analyses. In this research we present an analyses of seasonal trend variations of maximum and minimum temperature in Spanish conterminous land (1951-2010). Significance of trends is identified by using Man-Kendall test, and rate by Sen's approach. The results show no significant trend in winter and autumn in maximum and minimum temperature in recent decades. Spring maximum is not significant in recent decades but is significant in minimum; summer trends are not significant for the last decades. The rates of maximum and minimum for the last decades decrease, and minimum usually show highest value than maximum from the beginning of 90 's.
\end{abstract}

Key words: Temperatures, Trends, temporal windows, Spain. 


\section{INTRODUCCIÓN}

En toda serie de datos del clima su tendencia está condicionada por la longitud del periodo y la elección del año de inicio (Soon et al., 2004; Bladé y Castro Díaz, 2010; Liebmann et al., 2010; Lüdecke et al., 2011; Fyfe et al., 2013), lo que dificulta comparar las tasas publicadas en la bibliografía.

El análisis de tendencias de las temperaturas promedio mensuales de máximas (Tmax) y mínimas (Tmin) en la España peninsular con la malla de la base de datos MOTEDAS, ha permitido identificar una señal global positiva y significativa en el periodo 1951-2010 que concuerda con estudios previos, desigual en los valores de máximas y mínimas, distinta a lo largo de los meses y de magnitud heterogénea en el espacio; por último en las décadas recientes se ha reconocido la existencia del denominado hiato térmico (González-Hidalgo et al., 2015 a y b), definido como la ausencia de tendencia significativa (Meehl, 2015).

En el presente trabajo se ofrecen nuevos resultados sobre la evolución de las tendencias estacionales y anuales de las temperaturas de las décadas recientes en España peninsular, con especial énfasis en el efecto sobre la tendencia del periodo elegido y su longitud.

\section{BASE DE DATOS Y MÉTODO}

El análisis utiliza la base de datos de temperaturas promedio mensuales denominada MOTEDAS (Gonzalez-Hidalgo et al., 2015 a). Las series promedio estacionales y anuales de Tmax y Tmin se calcularon como promedio de las series mensuales de la malla de alta resolución $(10 \times 10 \mathrm{~km})$ obtenida a partir de 1358 observatorios, todos ellos libres de datos anómalos, de inhomogeneidades y reconstruidos sobre un total de 4710 series originales procedentes de los archivos de AEMet. Se acepta que la serie promedio es un buen descriptor del conjunto del territorio peninsular español dada la señal global homogénea de aumento térmico, si bien somos conscientes de sus variaciones espaciales actualmente investigadas.

El análisis de tendencias de estas series consta de dos fases: la significación se ha evaluado mediante la prueba de Mann-Kendal $(\mathrm{p}<0,05)$; la intensidad o tasa del periodo se ha calculado con el estadístico de Sen. El análisis se ha aplicado a todo el periodo aplicando ventanas temporales comprendidas entre 60 años (la serie completa) y 20 años, considerada la longitud mínima de una tendencia exenta de la variabilidad natural. Los resultados se centrarán especialmente en las ventanas de 30, 25 y 20 años y especialmente en el periodo final. Para un análisis global de las ventanas móviles véase Gonzalez-Hidalgo et al. (2015 b).

\section{RESULTADOS}

La evolución de los valores de la tasa promedio anual y estacional de Tmax y Tmin y su significación acorde los periodos de 20, 25 y 30 años (entre 1951 y 2010), se muestran en la Figura 1. En general a medida que la ventana de tiempo se amplía las diferencias entre periodos se suavizan en las cuatro estaciones y valores anuales, tanto en Tmax como Tmin, y viceversa las mayores variaciones entre periodos se encuentran en la ventana de 20 años. 

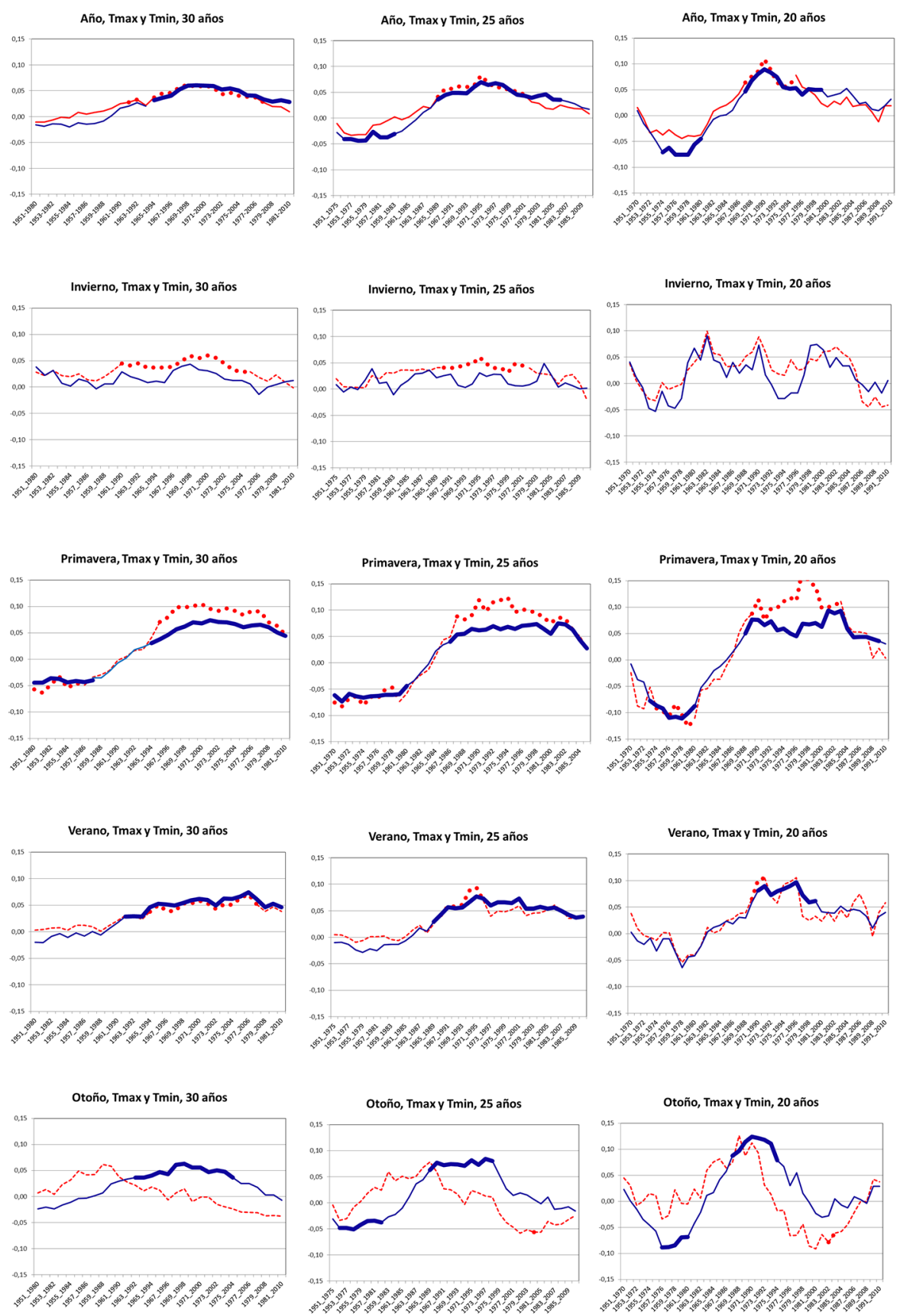

Figura 1. Evolución de las tasas anuales y estacionales de Tmax y Tmin en ventanas móviles de 30, 25 y 20 años. Línea discontinua (rojo) Tmax, línea continua (azul) Tmin. El grosor indica la significación $(p<0.05)$ según test de Mann-Kendal. Valores en ${ }^{\circ} \mathrm{C} /$ década según test de Sen. 


\begin{tabular}{|c|c|c|c|c|c|c|c|c|c|c|c|}
\hline & & Tmax & Tmin & Tmax & Tmin & Tmax & Tmin & Tmax & Tmin & Tmax & Tmin \\
\hline Periodo & $\mathrm{A}$ & \multicolumn{2}{|c|}{ Año } & \multicolumn{2}{|c|}{ Invierno } & \multicolumn{2}{|c|}{ Primavera } & \multicolumn{2}{|c|}{ Verano } & \multicolumn{2}{|c|}{ Otoño } \\
\hline $1970-1999$ & 30 & 0,057 & 0,060 & 0,055 & 0,033 & 0,101 & 0,070 & 0,053 & \begin{tabular}{|l|}
0,059 \\
\end{tabular} & $-0,010$ & 0,056 \\
\hline $1971-2000$ & 30 & 0,061 & 0,060 & 0,060 & 0,031 & 0,104 & 0,068 & 0,059 & 0,062 & $-0,001$ & 0,056 \\
\hline $1972-2001$ & 30 & 0,056 & 0,059 & 0,056 & 0,025 & 0,095 & 0,074 & 0,053 & 0,060 & $-0,001$ & 0,047 \\
\hline $1973-2002$ & 30 & 0,043 & 0,052 & 0,045 & 0,015 & 0,092 & 0,070 & 0,043 & 0,050 & $-0,015$ & 0,050 \\
\hline $1974-2003$ & 30 & 0,046 & 0,055 & 0,032 & 0,012 & 0,096 & 0,070 & 0,051 & \begin{tabular}{|l|}
0,062 \\
\end{tabular} & $-0,019$ & 0,047 \\
\hline $1975-2004$ & 30 & 0,041 & 0,050 & 0,029 & 0,012 & 0,092 & 0,067 & 0,051 & 0,062 & $-0,024$ & 0,036 \\
\hline $1976-2005$ & 30 & 0,039 & 0,041 & 0,029 & 0,006 & 0,083 & 0,061 & 0,064 & 0,066 & $-0,030$ & 0,025 \\
\hline $1977-2006$ & 30 & 0,037 & 0,040 & 0,018 & $-0,014$ & 0,092 & 0,064 & 0,071 & 0,074 & $-0,031$ & 0,025 \\
\hline $1978-2007$ & 30 & 0,027 & 0,033 & 0,011 & 0,000 & 0,090 & 0,066 & 0,051 & 0,061 & $-0,031$ & 0,018 \\
\hline $1979-2008$ & 30 & 0,020 & 0,029 & 0,023 & 0,005 & 0,070 & 0,061 & 0,038 & 0,046 & $-0,037$ & 0,003 \\
\hline $1980-2009$ & 30 & 0,019 & 0,031 & 0,010 & 0,010 & 0,063 & 0,051 & 0,047 & 0,052 & $-0,036$ & 0,003 \\
\hline $1981-2010$ & 30 & 0,010 & 0,028 & $-0,002$ & 0,012 & 0,046 & 0,044 & 0,038 & 0,046 & $-0,037$ & $-0,007$ \\
\hline $1970-1994$ & 25 & 0,063 & 0,060 & 0,048 & 0,010 & 0,090 & 0,065 & 0,088 & 0,066 & $-0,003$ & 0,071 \\
\hline 1971-1995 & 25 & 0,082 & 0,069 & 0,060 & 0,031 & 0,119 & 0,061 & 0,094 & $\mathbf{0 , 0 7 7}$ & 0,023 & 0,082 \\
\hline $1972-1996$ & 25 & 0,068 & 0,064 & 0,048 & 0,024 & 0,097 & 0,063 & 0,069 & \begin{tabular}{|l|}
0,073 \\
\end{tabular} & 0,019 & 0,073 \\
\hline $1973-1997$ & 25 & 0,059 & 0,068 & 0,040 & 0,028 & 0,115 & 0,069 & 0,040 & \begin{tabular}{|l|}
0,060 \\
\end{tabular} & 0,013 & 0,084 \\
\hline $1974-1998$ & 25 & 0,060 & 0,064 & 0,040 & 0,028 & 0,119 & 0,063 & 0,050 & 0,066 & 0,011 & 0,080 \\
\hline $1975-1999$ & 25 & 0,052 & 0,054 & 0,032 & 0,010 & 0,124 & 0,069 & 0,048 & 0,066 & $-0,020$ & 0,054 \\
\hline $1976-2000$ & 25 & 0,052 & 0,045 & 0,049 & 0,006 & 0,096 & 0,065 & 0,052 & 0,064 & $-0,038$ & 0,027 \\
\hline $1977-2001$ & 25 & 0,045 & 0,043 & 0,044 & 0,006 & 0,102 & 0,070 & 0,059 & 0,073 & $-0,047$ & 0,014 \\
\hline $1978-2002$ & 25 & 0,031 & 0,040 & 0,040 & 0,009 & 0,099 & 0,071 & 0,041 & 0,054 & $-0,059$ & 0,019 \\
\hline 1979-2003 & 25 & 0,029 & 0,044 & 0,030 & 0,015 & 0,095 & 0,074 & 0,046 & 0,054 & $-0,051$ & 0,015 \\
\hline 1980-2004 & 25 & 0,019 & 0,046 & 0,029 & 0,049 & 0,085 & 0,064 & 0,046 & $\mathbf{0 , 0 5 7}$ & $-0,056$ & 0,006 \\
\hline $1981-2005$ & 25 & 0,017 & 0,036 & 0,027 & 0,028 & 0,075 & 0,055 & 0,051 & 0,054 & $-0,056$ & $-0,002$ \\
\hline $1982-2006$ & 25 & 0,025 & 0,036 & 0,010 & 0,003 & 0,086 & 0,075 & 0,061 & 0,056 & $-0,035$ & 0,011 \\
\hline $1983-2007$ & 25 & 0,021 & 0,032 & 0,025 & 0,012 & 0,084 & 0,073 & 0,051 & 0,050 & $-0,042$ & $-0,013$ \\
\hline $1984-2008$ & 25 & 0,019 & 0,028 & 0,028 & 0,006 & 0,062 & 0,063 & 0,036 & \begin{tabular}{|l|}
0,042 \\
\end{tabular} & $-0,041$ & $-0,011$ \\
\hline $1985-2009$ & 25 & 0,018 & 0,020 & 0,011 & 0,001 & 0,051 & 0,043 & 0,036 & \begin{tabular}{|l|}
0,037 \\
\end{tabular} & $-0,032$ & $-0,007$ \\
\hline 1986-2010 & 25 & 0,008 & 0,017 & $-0,020$ & 0,002 & 0,027 & $\mathbf{0 , 0 2 7}$ & 0,037 & \begin{tabular}{|l|}
0,039 \\
\end{tabular} & $-0,024$ & $-0,016$ \\
\hline $1970-1989$ & 20 & 0,085 & 0,080 & 0,060 & 0,026 & 0,088 & $\mathbf{0 , 0 7 7}$ & 0,065 & 0,059 & 0,087 & 0,114 \\
\hline $1971-1990$ & 20 & 0,109 & 0,089 & 0,089 & 0,073 & 0,114 & 0,076 & 0,101 & 0,081 & 0,112 & 0,124 \\
\hline $1972-1991$ & 20 & 0,089 & 0,084 & 0,060 & 0,016 & 0,075 & 0,066 & 0,104 & 0,090 & 0,093 & 0,121 \\
\hline 1973-1992 & 20 & 0,065 & 0,075 & 0,026 & $-0,003$ & 0,097 & 0,073 & 0,071 & 0,072 & 0,031 & 0,118 \\
\hline 1974-1993 & 20 & 0,055 & 0,055 & 0,018 & $-0,029$ & 0,099 & 0,056 & 0,057 & 0,080 & 0,013 & 0,111 \\
\hline $1975-1994$ & 20 & 0,059 & 0,052 & 0,015 & $-0,029$ & 0,111 & 0,060 & 0,093 & 0,084 & $-0,018$ & 0,079 \\
\hline 1976-1995 & 20 & 0,079 & 0,054 & 0,045 & $-0,018$ & 0,118 & 0,050 & 0,098 & \begin{tabular}{|l|}
0,090 \\
\end{tabular} & $-0,017$ & 0,067 \\
\hline $1977-1996$ & 20 & 0,055 & 0,040 & 0,026 & $-0,018$ & 0,112 & 0,045 & 0,105 & 0,097 & $-0,065$ & 0,030 \\
\hline 1978-1997 & 20 & 0,050 & 0,051 & 0,027 & 0,015 & 0,173 & 0,069 & 0,033 & \begin{tabular}{|l|}
0,072 \\
\end{tabular} & $-0,065$ & 0,055 \\
\hline 1979-1998 & 20 & 0,040 & 0,050 & 0,046 & 0,072 & 0,146 & 0,067 & 0,025 & 0,059 & $-0,044$ & 0,015 \\
\hline 1980-1999 & 20 & 0,024 & 0,050 & 0,043 & 0,074 & 0,140 & 0,070 & 0,033 & 0,061 & $-0,086$ & $-0,002$ \\
\hline $1981-2000$ & 20 & 0,017 & 0,037 & 0,060 & 0,063 & 0,098 & 0,062 & 0,024 & 0,042 & $-0,091$ & $-0,023$ \\
\hline $1982-2001$ & 20 & 0,028 & 0,040 & 0,062 & 0,030 & 0,100 & 0,093 & 0,041 & 0,040 & $-0,064$ & $-0,031$ \\
\hline 1983-2002 & 20 & 0,022 & 0,044 & 0,069 & 0,049 & 0,104 & 0,088 & 0,024 & 0,039 & $-0,078$ & $-0,028$ \\
\hline $1984-2003$ & 20 & 0,036 & 0,053 & 0,057 & 0,033 & 0,111 & 0,093 & 0,045 & 0,052 & $-0,061$ & 0,005 \\
\hline $1985-2004$ & 20 & 0,018 & 0,039 & 0,048 & 0,033 & 0,066 & 0,058 & 0,030 & 0,042 & $-0,058$ & $-0,007$ \\
\hline 1986-2005 & 20 & 0,020 & 0,023 & 0,022 & 0,007 & 0,053 & 0,043 & 0,060 & 0,046 & $-0,045$ & $-0,013$ \\
\hline $1987-2006$ & 20 & 0,021 & 0,026 & $-0,035$ & $-0,002$ & 0,053 & 0,043 & 0,075 & 0,043 & $-0,021$ & 0,009 \\
\hline $1988-2007$ & 20 & 0,007 & 0,012 & $-0,044$ & $-0,016$ & 0,050 & 0,043 & 0,047 & 0,033 & $-0,001$ & 0,004 \\
\hline 1989-2008 & 20 & $-0,012$ & 0,010 & $-0,025$ & 0,002 & 0,003 & 0,039 & $-0,005$ & 0,011 & $-0,001$ & $-0,004$ \\
\hline 1990-2009 & 20 & 0,019 & 0,019 & $-0,044$ & $-0,018$ & 0,022 & 0,036 & 0,040 & 0,032 & 0,042 & 0,029 \\
\hline 1991-2010 & 20 & 0,019 & 0,032 & $-0,041$ & 0,006 & 0,003 & 0,030 & 0,058 & 0,040 & 0,038 & 0,029 \\
\hline
\end{tabular}

Tabla 1. Magnitud de la tendencia en ventanas móviles de 30, 25 y 20 años de los promedios anuales y estacionales de Tmax y Tmin. En negrita significación $p<0.05$. Valores en ${ }^{\circ} \mathrm{C} / a n ̃ o . A$ Intervalo de años de la ventana analizada 
El estudio de la evolución de las tasas en los tres intervalos seleccionados permite observar que tanto Tmax como Tmin alcanzaron unos máximos en décadas pasadas y la intensidad y significación del aumento térmico desciende durante las décadas finales. Este descenso difiere según series climáticas y medidas termométricas pero puede concretarse su máxima intensidad en torno a 1970-1990. En la Tabla 1 se muestran los valores del estadístico de Sen (en ${ }^{\circ} \mathrm{C} / \mathrm{año}$ ) de las series de promedios anuales y estacionales de Tmax y Tmin y su significación en las ventanas de 30, 25 y 20 años. Los valores se refieren a las ventanas que comienzan en el año 1970.

En la ventana de 30 años y en las series promedio anuales de Tmax y Tmin destaca la ausencia de significación desde 1979-2008 de Tmax, con la consecuencia de Tmin $>$ Tmax. Estacionalmente el invierno no ha sido significativo en Tmin, así como Tmax deja de serlo desde la ventana 1975-2004. La primavera es la estación con tendencias significativas mantenidas, y tasas superiores en Tmax respecto a Tmin; en Tmax no es significativa desde 1979-2008 y de nuevo Tmin>tmax; por último en otoño las tendencias no son significativas tanto en Tmax (con signo negativo) como Tmin (esta desde 1976-2005).

El cambio de ventana a 25 años descubre nuevos hechos. Los promedios anuales de Tmax y Tmin dejan de ser significativos en las ventanas que comienzan en 1978 y 1983 respectivamente. Estacionalmente las tendencias de Tmin del invierno no son significativas y en Tmax dejan de serlo desde 1979-2003; en primavera las tendencias de Tmax no son significativas desde la ventana que comienza en 1985-2009, manteniendo la significación las de Tmin, la ventaja de las tasas de Tmax respecto a Tmin se aminora en las ventanas finales. En verano Tmax deja de ser significativa desde 19731997, mientras que Tmin mantiene su significación si bien desciende en intensidad. Por último en otoño Tmax no es significativa y Tmin deja de serlo desde la ventana 1975-1999, tras presentan un periodo de fuerte ascenso significativo en las ventanas del inicio de la década de los años setenta

La ventana más corta de 20 años permite precisar finalmente hechos que el análisis de periodos más largos oculta. Los promedios anuales de Tmax y Tmin han dejado de tener una tendencia significativa desde las ventanas 1977-1996 y 1981-2000 respectivamente, lo que implica que en las décadas finales las tasas promedio anuales de Tmin superaron las de Tmax. Estacionalmente en invierno las tasa de Tmax y Tmin no presenta significación salvo en ventanas aisladas; en primavera las tasas de Tmin han mantenido su significación aunque descendiendo su intensidad, y las tasas de Tmax en su descenso dejan de tener significación desde la ventana 1985-2004; tampoco es significativa la secuencia de ventanas móviles de Tmax en verano desde 1973-1992, y de Tmin desde 1981-2000; por último las tasas de otoño de Tmax no han sido significativas excepto el conjunto de ventanas en torno al inicio de los setenta en Tmin.

\section{DISCUSIÓN}

\subsection{Aspectos generales}

Diversos estudios han sugerido que durante la segunda mitad del XX la evolución térmica en España peninsular ha dependido de la primavera y verano, y especialmente de la evolución de Tmax (periodo 1971-2000, Brunet et al., 2007; periodo 1961-2002, del Rio et al., 2011, 2012; periodo 1961-2010, Rios et al., 2012; periodo 1981-2010, 
Llorente, 2012; periodo 1951-2012, Guijarro, 2013; periodo 1976-2005, en Portugal, Ramos et al., 2011). En general las tasas de promedios anuales de los citados estudios concuerdan groso modo con las del presente análisis en los periodos equivalentes de MOTEDAS (no mostradas), así como existe coincidencia en la identificación del máximo estacional de verano y primavera, pero nuestros resultados sugieren que el calentamiento más reciente de España peninsular ha estado más controlado por la temperatura nocturna (Tmin) que la diurna (Tmax).

El análisis de ventanas móviles ha permitido identificar que el máximo aumento de temperatura en España peninsular ha ocurrido en torno a 1970-1990, periodo tras el cual la magnitud de las tasas desciende y pierde significación excepto Tmin de primavera. En los momentos finales de MOTEDAS las tasas, salvo en invierno y otoño, siguen manteniendo en general un signo positivo, pero han perdido significación estadística. Estos resultados demuestran la existencia del parón térmico en España peninsular, que se adelanta una década respecto al año 2000, la fecha aceptada comúnmente. De este modo se puede afirmar que durante los 20-25 años más recientes la tendencia de las temperaturas ha dejado de ser significativa en España, exceptuando Tmin de primavera.

\subsection{Las tendencias de Tmax y Tmin}

Durante el siglo XX se ha señalado un aumento térmico superior en la temperatura nocturna que diurna (i.e. Tmin $>$ Tmax), si bien desde 1980 los resultados difieren entre autores (Cohen et al., 2013; Makowsky et al., 2008; Vose et al., 2005). Las razones de esta discrepancia son variadas y se han relacionado con la densidad de información de las bases de datos (Janis et al., 2004; McNider et al., 2010), la calidad de las series (Fall et al., 2011), y sobre todo los diferentes factores que afectan ambas mediciones.

Los registros de Tmax y Tmin reflejan las condiciones diurnas y nocturnas, y el efecto que los diferentes factores que las controlan ejercen sobre ellas (Mahmood et al., 2010); en general se acepta que factores de carácter general afectan las medidas de Tmax, y que los factores locales controlan Tmin. Entre los primeros se citan la radiación solar, modulada por la nubosidad, las emisiones y el vapor de agua (Dai et al., 1999; Philipona et al., 2005; Makoswsky et al., 2008; Lauritsen y Rogers, 2012), mientras los factores que rigen Tmin se relacionan sobre todo con factores locales porque durante la noche el registro de la temperatura refleja en realidad la de la capa de aire más cercana a la superficie, en donde los flujos de calor latente y sensible pueden estar modificados localmente por cambios de uso del suelo (Karl et al., 1988; Kalnay y Cai, 2003; Pielke et al., 2007; Christy et al., 2009; Kloztbach et al., 2009).

Philipona et al. (2005) en un estudio paneuropeo identificaron un enfriamiento reciente en los promedios de temperatura de la península Ibérica excepto al sureste (1995-2004), y un fuerte descenso de la humedad específica en todo el continente en un gradiente oeste-este. Los hechos citados sugerirían que la ausencia de vapor de agua pudiera ser la causa del enfriamiento, en coincidencia con los resultados de Vicente-Serrano et al. (2014a, 2014b), que han sugerido que el aumento de la evapotranspiración potencial en la Península Ibérica, por el aumento de temperatura, no ha producido un aumento del vapor de agua en la atmósfera probablemente por la 
modificaciones de los patrones de la circulación atmosférica y oceánica. La ausencia de vapor de agua sobre la Península Ibérica, además coincide con el aumento de las horas de insolación y el paralelo descenso de la nubosidad (Sanchez-Lorenzo et al., 2009, 2012), sincrónicos a la tendencia negativa (aunque no significativa) de las precipitaciones anuales (de Luis et al, 2010). En resumen: el aumento de la insolación y reducción de la nubosidad en la Península Ibérica, el aumento de la evapotranspiración potencial por el aumento térmico, la ausencia de modificaciones en la humedad específica y el descenso de la humedad relativa sin cambios apreciables en la precipitación, sugerirían en su conjunto que la transferencia de humedad desde los océanos no habría tenido lugar. Esta cadena de argumentos pudiera poder convertir a los bajos contenidos de vapor atmosférico como uno de los principales argumentos capaces de explicar las tendencias de Tmax durante las últimas décadas, y concuerdan con los efectos de dicho gas sobre las temperaturas diurnas (Dai et al., 1999; Philipona et al., 2005).

La evolución de Tmin no puede argumentarse del mismo modo, pues el efecto del vapor de agua sobre ellas es el inverso a Tmax (Philipona et al., 2005); de hecho solamente se ha detectado un aumento de humedad en primavera, en cuyo caso la retroalimentación ha podido ser abortada por falta de suministros desde el océano, coincidiendo con las tendencias negativas de precipitación (en este caso sí significativas) especialmente en marzo (Gonzalez-Hidalgo et al., 2011).

Un comentario final se refiere a los eventuales efectos de los factores locales ante las profundas modificaciones de los paisajes españoles durante las décadas recientes. Estas modificaciones incluyen transformaciones en nuevos regadíos (Ministerio de Agricultura, Pesca y Alimentación, 2009), infraestructuras, urbanización (Zúñiga et al., 2012), e incendios forestales entre otros (Enríquez y del Moral, 2012). Estos cambios masivos de usos del suelo durante los últimos 30 años son consistentes con algunos de los factores que alteran las temperaturas nocturnas, como el regadío cuyos efectos positivos sobre las temperaturas nocturnas ha sido identificado sobre todo en las estaciones secas y cálidas (Dai et al., 1999; Gallo et al., 1999; Karl et al., 1993; Christy et al., 2006). Un Segundo cambio ambiental localmente muy intense ocurrido en España es la urbanización, incluyendo como tal todo tipo de uso del suelo artificial. El debate sobre sus efectos es permanente, pero allí donde se ha reconocido se sugiere un efecto positivo sobre Tmin (Karl et al., 1988) cuya importancia se ha llegado a evaluar en un 50\% del aumento térmico total (Kalnay y Cai, 2003).

Las ideas precedentes son reflexiones iniciales actualmente en estudio y no podemos concretar más, salvo que las zonas donde se ha producido un incremento mayor de nuevos regadíos y la transformación de los paisajes ha sido máxima (costa de levante y mitad sur peninsular grosso modo), tienen tasas de aumento de Tmin en los niveles más elevados.

En resumen, la evolución de las temperaturas en España está lejos de ser completamente comprendida y permanecen sin resolver diversos interrogantes que incluyen la explicación del diferente reparto espacial de la magnitudes de sus tasas.

\section{CONCLUSIONES}

El análisis de las tendencias de las temperaturas en ventanas móviles corrobora el efecto que tiene la longitud del periodo elegido y el año de inicio en la significación 
y magnitud de aquella, y permite apreciar que el aumento de temperaturas de la segunda mitad del siglo XX en España peninsular ha dependido del comportamiento de primavera y verano, sobre todo de los valores de sus temperaturas mínimas, y que el aumento se ha producido especialmente entre los años 1970 y 1990. Durante los 20-25 años más recientes las tendencias han dejado de ser significativas excepto en Tmin de primavera. Factores globales ligados a la circulación general parecen relacionarse con el comportamiento de Tmax, mientras que existen evidencias de que el comportamiento de Tmin pudiera estar más relacionado con factores locales.

\section{AGRADECIMIENTOS}

Ministerio de Ciencia e Innovación, Gobierno de España, Proyecto DESEMON, CGL2014-52135-C3-3-R. Gobierno Regional de Aragón DGA-FSE (Grupo de Investigación Consolidado "Clima, Agua, Cambio Global y Sistemas Naturales"). Dhais Peña-Angulo es becaria de doctorado del Programa FPI del Ministerio de Ciencia e Innovación. Datos climáticos originales cedidos por AEMet.

\section{REFERENCIAS}

Bladé I, Castro-Díez Y. (2010). Tendencias atmosféricas en la Península ibérica durante el periodo instrumental en el contexto de la variabilidad climática. En Perez F, Boscolo R (eds). Clima en España: pasado, presente y futuro. MedCLIVAR: Madrid, 25-42.

Brunet M, Jones P, Sigro J, Saladie O, Aguilar E, Moberg A, Della-Marta PM, Lister D, Walther A, Lopez D. (2007). Temporal and spatial temperature variability and change over Spain during 1850-2005. Journal of Geophysical Research 112: D12117.

Christy JR, Norris W, Redmond K, Gallo KP. (2006). Methodology and results of calculating central California surface temperature trends: evidence of humaninduced climate change? Journal of Climate 10: 548-563.

Christy JR, Norris WB, McNider RT. (2009). Surface temperature variations in East Africa and possible causes. Journal of Climate 22: 3342-3356.

Cohen JL, Furtado JC, Barlow M, Alexeev VA, Cherry JE. (2013). Asymmetric seasonal temperature trends. Geophysical Research Letter 39: L04705, DOI: 10.1029/2011GL050582.

Dai A, Trenberth KE, Karl T. (1999). Effects of clouds, soil moisture, precipitation, and water vapour on diurnal temperature range. Journal of Climate 12: 24512473.

de Luis M, Brunetti M, Gonzalez-Hidalgo JC, Longares LA, Martín-Vide J. (2010). Changes in seasonal precipitation in the Iberian Peninsula during 1946-2005. Global Planetary Changes 74: 27-33.

del Rio S, Herrero L, Pinto-Gomes C, Penas A. (2011). Spatial analyses of mean temperature trends in Spain over the period 1961-2006. Global Planetary Change 78: $65-75$. 
del Rio S, Cano-Ortiz A, Herrero L, Penas A. (2012). Recent trends in mean maximum and minimum air temperatures over Spain (1961-2006). Theoretical and Applied Climatology 109: 605-626.

Enríquez E, Del Moral L. (2012). Los Incendios Forestales en España. Decenio 20012010. Ministerio de Agricultura, Alimentación y Medio Ambiente. Madrid.

Fall S, Watts A, Nielsen-Gammon J, Jones E, Niyogi D, Christy JR, Pielke Sr RA. (2011). Analysis of the impacts of station exposure on the US Historical Climatology Network temperatures and temperature trends. Journal of Geophysical ResearchAtmospheres 116, D14120, Doi:10.1029/2010JDO15146.

Fyfe JC, Gillett NP, Zwiers FW. (2013). Overestimated global warming over the past 20 years. Nature Climate Change 3: 767-769.

Gallo KP. (2005). Evaluation of temperature differences for paired stations of the US climate reference network. Journal of Climate 18: 1629-1636.

Gonzalez-Hidalgo JC, Peña-Angulo D, Brunetti M, Cortesi N. (2015 a). MOTEDAS: a new monthly temperature database of Spanish continental land and the temperature trend between 1951-2010. International Journal of Climatology, 35: 4444-4463 DOI: 10.1002/joc.4298 (on line).

Gonzalez-Hidalgo JC, Peña-Angulo D, Brunetti M, Cortesi C. (2015 b). Recent trend in temperature evolution in Spanish mainland (1951-2010): from warming to hiatus. International Journal of Climatology doi: 10.1002/joc.4519.

Guijarro JA. (2013). Tendencias de la Temperatura. In Garcia-Legaz C, Valero F (eds). Fenómenos meteorológicos adversos en España, WCRP, AMV Ediciones: Madrid; 313-323.

Janis MJ, Hubbard KG, Redmond KT. (2004). Station density strategy for monitoring long-term climatic changes in the contiguous United States. Journal of Climate 17: 151-162.

Kalnay E, Cai M. (2003). The impact of urbanization and land-use change on climate. Nature 423: 528-531.

Karl TR, Diaz HF, Kukla G. (1988). Urbanization: its detection and effects in the United Stated climate records. Journal of Climate 1: 1099-1123.

Karl TR, Jones PD, Knight RW, Kukla G, Plummer N, Razuvayev V, Gallo KP. Lindseay J, Charlson RJ, Peterson TC. (1993). Asymmetric trends of daily maximum and minimum temperature. Bulletin American Meteorological Society 74: 1007-1023.

Klotzbach PJ, Pielke RA Sr, Pielke RA Jr, Christy JR, McNider RT. (2009). An alternative explanation for differential temperature trends at the surface and the lower troposphere. Journal of Geophysical Research 114: D21102, doi:10.1029/2009JD011841.

Lauritsen RG, Rogers JC. (2012). U.S. Diurnal Temperature Range Variability and Regional Causal Mechanisms, 1901-2002. Journal of Climate 25: 7216-7231.

Liebmann B, Dole RM, Jones C, Bladé I, Allured D. (2010). Influence of choice of time on global surface temperature trend estimated. Bulletin American Meteorological Society 91: 1485-149. 
Llorente M. (2012). Tendencias españolas de variables agrometeorológicas en los últimos 30 años. Departamento de Producción Vegetal, Universidad Politécnica de Madrid, ETSIA, 99 p.

Lüdecke HJ, Link R, Ewert FK. (2011). How natural is the recent centennial warming? An Analysis of 2249 surface temperature records. Journal of Modern Physics 22: 10, doi:10.1142/SO1291831111016798.

Mahmood R, Pielke RA Sr, Hubbard KG, et al. (2010). Impact of land use/land cover change on climate and future research priorities. Bulletin American Meteorological Society pp.37-46.

Makowsky K, Wild M, Ohmura A. (2008). Diurnal temperature range over Europe between 1950-2005. Atmospheric Chemistry and Physics 8: 6483-6498.

McNider RT, Christy JR, Biazar A. (2010). A Stable Boundary Layer Perspective on Global Temperature Trends. International Conference on Planetary Boundary Layer and Climate Change, Cape Town, SA 13, Number: 012003, DOI: 10.1088/1755-1315/13/1/012003.

Meehl GA. (2015). Decadal climate variability and the early-2000s hiatus. US Clivar Variations 13: 1-6.

Ministerio de Agricultura, Pesca y Alimentación. (2009). Encuesta sobre superficies y rendimientos de cultivos. Informe sobre regadíos en España. Publicaciones de la Secretaría General Técnica, Subdirección General de Estadística. Madrid.

Pielke RA, Davey CA, Niyogi D, Fall S, et al. (2007). Unresolved issues with the assessment of multidecadal global land Surface temperature trends. Journal of Geophysical Research 112: D24S08, Doi:10.1029/2006JDD008229.

Philipona R, Dürr B, Ohmura A, Ruckstuhl C. (2005). Anthropogenic greenhouse forcing and strong water vapour feedback increase temperature in Europe. Geophysical Research Letter 32, L19809, doi:10.1029/2005GL023624, 2005

Ramos AM, Trigo RM, Santo FE. (2011). Evolution of extreme temperatures over Portugal: recent changes and future scenarios. Climate Research 48: 177-192.

Rios D, Penas D, del Rio S. (2012). Comparative analysis of mean temperature trends in continental Spain over the period 1961-2010. International Journal of Geobotanical Research 2: 41-55.

Sanchez-Lorenzo A, Calbó J, Brunetti M, Deser C. (2009). Dimming/brightening over the Iberian Peninsula: Trends in sunshine duration and cloud cover and their relations with atmospheric circulation. Journal of Geophysical Research Atmosphere: 114, D00D09, doi:10.1029/2008JD011394.

Sanchez-Lorenzo A, Calbó J, Wild M. (2012). Increasing cloud cover in the 20th century: review and new findings in Spain. Climate of the Past 8: 1199-1212.

Soon WH, Legates DR, Baliunas SL. (2004). Estimation and representation of long-term ( $>40$ year) trends of Northern-Hemisphere-gridded surface temperature: A note of caution. Geophysical Research Letter 31: L03209, doi:10.1029/2003GL019141.

Vicente-Serrano SV, Azorin-Molina C, Sanchez-Lorenzo A, et al. (2014a). Temporal evolution of surface humidity in Spain: recent trends and possible physical mechanisms. Climate Dynamic 42: 2655-2674. 
Vicente-Serrano S, Azorin-Molina C, Sanchez-Lorenzo A, Revuelto J, et al. (2014b). Reference evapotranspiration variability and trends in Spain, 1961-2011. Global and Planetary Change 121: 26-40.

Vose RS, Easterling DR, Gleason B. 2005. Maximum and minimum temperature trends for the globe: an uptodate through (2004). Geophysical Research Letter 32: L23822.

Zúñiga M, Pueyo A, Calvo JL. (2012). The Spanish population during the 20th century and beyond. Journal of Maps 8: 1-6. 